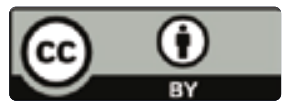

\title{
FACTORES DE RIESGO ASOCIADOS A LA RETINOPATÍA DE LA PREMATURIDAD
}

\author{
RISK FACTORS ASSOCIATED WITH RETINOPATHY OF PREMATURITY
}

\author{
Ramos-Uribe, Rebeca ${ }^{1, a}$ \\ 1. Médico del Hospital Félix Torrealva \\ a. Médico Pediatra
}

Correspondencia:

Ramos Uribe, Rebeca

Número de celular: 994762165

Correo Electrónico:

relvy_ru@outlook.com

Contribuciones De Autoría:

RRU participó en el diseño del estudio, el análisis de los datos, revisión crítica del artículo y aprobó la versión final.

Conflicto De Intereses: No declarados.

Financiamiento: Autofinanciado.

\section{Como Citar}

Ramos Uribe Rebeca. Factores de riesgo asociados a la retinopatía de la prematuridad. Rev méd panacea. 2019; 8(3): 108-115. DOI: https://doi.org/10.35563/rmp.v8 i3.194

Recibido: 17 - 08 - 2019

Aceptado: 20 - 09 - 2019

Publicado: 22 - 09 - 2019

\section{RESUMEN}

Objetivo: Describir los factores de riesgo asociados a la a la retinopatía de la prematuridad. Materiales y métodos: Estudio de revisión, descriptivo transversal. Resultados: La retinopatía de la prematuridad (ROP) es una enfermedad reportada a nivel mundial en los bebés prematuros en los que se afecta el desarrollo de la vasculatura retiniana. La presentación varía desde casos leves que pueden llegar a resolverse como casos graves que culminan en ceguera. Los factores de riesgo principales son el bajo peso al nacer y la edad gestacional, pero también se han descritos otros como la transfusión de sangre, la terapia con oxígeno, entre otros. Discusión: Se ha reportado una incidencia entre 19 a 30\% a nivel mundial en bebés prematuros con un rango de peso igual o menor a 1300-1500 gramos con 33 semanas de gestación. Sin embargo aún está en discusión si el sexo femenino o masculino estaría asociado a su desarrollo. Existen factores del manejo del recién nacido prematuro como la terapia prolongada con oxígeno, así como variables maternas clínicas y socioeconómicas y factores neonatales que influirían en la presentación de ROP. Conclusiones: Los principales factores de riesgo de la retinopatía neonatal bajo peso al nacer y con baja edad gestacional. Es necesario que a nivel local se genere evidencia cuantitativa sobre los factores de riesgo asociados a ROP en nuestras condiciones, con el objetivo de formular recomendaciones que apoyen a la parte clínica.

Palabras clave: Retinopatía de la prematuridad, factores de riesgo.

\section{ABSTRACT}

Objective: To describe the risk factors associated with retinopathy of prematurity. Materials and methods: Review study, cross-sectional descriptive. Results: Retinopathy of prematurity (ROP) is a disease reported worldwide in premature babies in which it refers to the development of the retinal vasculature. The current presentation of cases that can be resolved as cases of tombs that culminate in blindness. The main risk factors are, among others, the administration of age and gestational age. Discussion: An incidence of 19 to $30 \%$ worldwide has been reported in premature babies with a weight range equal to or less than 1300-1500 grams in 33 weeks of gestation associated with its development. There are factors of management of premature newborns such as prolonged therapy with oxygen, as well as variables of clinical and socioeconomic variables and neonatal factors that influence the presentation of ROP. Conclusions: The main risk factors of neonatal retinopathy underweight at birth and with low gestational age. It is necessary that a local level is generated quantitative evidence on the risk factors associated with ROP in our conditions, with the aim of making recommendations that support the clinical part.

Keywords: retinopathy of prematurity, risk factors. 


\section{INTRODUCCIÓN}

La retinopatía de la prematuridad (ROP), también conocida como fibroplasia retrolental, es una patología neovascular, es decir, el desarrollo anormal de los vasos sanguíneos en la retina, comenzando los primeros días de vida, pudiendo progresar a su forma más severa que ocasiona la ceguera en semanas (1). Sin embargo, a pesar de ser una de las causas de ceguera más prevenible a nivel mundial, la retinopatía de la prematuridad se desarrolla hasta en $84 \%$ de los recién nacidos prematuros de menos de $1250 \mathrm{~g}$ de peso al nacer (2).

Las cifras actuales mencionan que actualmente existen 1,4 millones de niños ciegos en el mundo, correspondiendo unos 400 niños ciegos por año a consecuencia de la retinopatía de la prematuridad. Debido a los avances de la neonatología, la sobrevivencia de niños nacidos con 25 semanas de gestación y 700 gramos de peso ha incrementado en un $50 \%$ y es justamente en este grupo de pacientes donde la retinopatía de la prematuridad constituye un gran riesgo de ser padecida (3).

El riesgo de la retinopatía de la prematuridad es inversamente proporcional a la edad gestacional y al peso al nacer. Se han realizado varios estudios para dilucidar los factores de riesgos y todos identificaron la prematuridad y el bajo peso al nacer como los más relevantes, seguidos de las fluctuaciones en los niveles de oxígeno tomados por vía trans-cutánea, la asfixia-hipoxia, la acidosis, la exposición a la luz, el déficit de vitamina E y la septicemia, la ventilación mecánica, las transfusiones sanguíneas, el embarazo múltiple, las hemorragias y las apneas severas, entre otras (4).

Teniendo en cuenta esta realidad, se considera necesario realizar la revision bibliográfica de los factores de riesgo asociados a retinopatía de la prematuridad con el objetivo de tener information actualizada que permita una mayor discusión de los casos a nivel local.

\section{OBJETIVO GENERAL}

Describir y entender los factores de riesgo asociados a la retinopatía de la prematuridad.

\section{MATERIALES Y MÉTODOS}

Estudio descriptivo transversal de revisión científica, que empleó palabras clave como retinopatía, prematuridad, neonatología en los buscadores Google Scholar, PubMed y Scielo para obtener información actual referente a los factores de riesgo asociados a la retinopatía de la prematuridad.

\section{DEFINICIÓN}

La retinopatía del prematuro o de la prematuridad (ROP), se describió por primera vez en 1942, denominándola como fibroplasia retrolental, y en 1980 se le conoce como retinopatía del prematuro $(5,6,7)$. ROP es una enfermedad neovascular que afecta el desarrollo de los vasos retinianos de los prematuros por causas aún desconocidas, aunque mayormente relacionada al bajo peso al nacer y a la baja edad gestacional (8).

ROP es una enfermedad retiniana y del vítreo, vasoproliferativa y multifactorial, en la que los vasos sanguíneos retinianos dejan de crecer y desarrollarse. En algunos casos se cura completamente, sin embargo, también pueden quedar secuelas como la miopía. La enfermedad comienza en los primeros días de vida, pudiendo progresar rápidamente y causar ceguera en cuestión de semanas por desprendimiento de la retina (9).

La forma de presentación aguda se produce por interrupción de la vascularización normal de los vasos de la retina y por lo general el $90 \%$ de estos casos entran en regresión espontánea y se resuelven con una reacción cicatrizal mínima. Por otro lado, en la presentación crónica ocurre la proliferación de las membranas hacia el vítreo, provocando desprendimiento de la retina y cicatrización de la mácula, pudiendo llegar a la ceguera $(7,9)$.

\section{FISIOPATOLOGIA}

ROP es considerada de etiología multifactorial y de una fisiopatología compleja, siendo determinante la severidad de la prematuridad. Luego del nacimiento, la retina inmadura del bebé prematuro puede desarrollarse normalmente alcanzando la madurez retinal sin ningún problema. Sin embargo, en algunos casos, al nacer un bebé prematuro su inmadurez pulmonar y la consecuente carencia de oxígeno puede inhibir el estímulo de crecimiento de los vasos retinales, ocurriendo un desequilibrio entre la cantidad de vasos a irrigar y la cantidad de vasos desarrollados, formando dos zonas, una zona de retina isquémica o avascular y otra zona de retina vascularizada. Este fenómeno produce la liberación de sustancias vasoproliferativas (VEGF-factor de crecimiento endotelial) que llevan a la liberación de proteínas y si continúa el proceso ya hay crecimiento fibrovascular y de nuevos vasos (neovasos) que crecen desorganizadamente; en vez de ir horizontalmente en la superficie de la retina, toman una dirección vertical hacia el interior del ojo, hacia el vítreo. A este fenómeno se le conoce como microangiopatía vasoproliferativa $(10,11,12)$ 
Figura 1. Retinopatía del prematuro. Figura tomada y adaptada al español de la referencia 10.

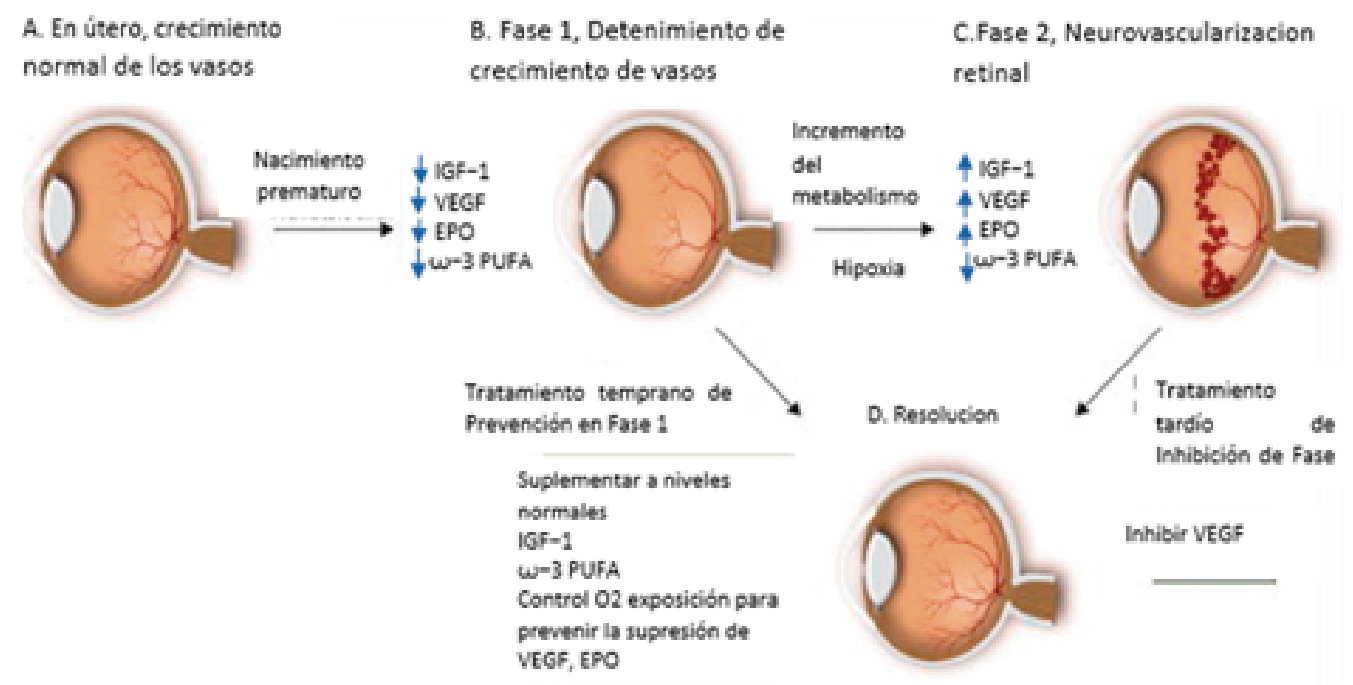

(A) En el útero la tensión de oxigeno es baja con un crecimiento vascular normal. (B) Corresponde a la Fase 1, después del nacimiento, se inhibe la vascularización de la retina a causa de la hiperoxia, perdida de nutrientes y factores de crecimiento del interfaz materno fetal. Al detenerse la angiogénesis y no proveer adecuadamente el desarrollo metabólico de la retina, se produce la hipoxia. (C) Fase 2, la hipoxia en la retina estimula la expresión de eritropoyetina (EPO) y el factor de crecimiento endotelial vascular (VEGF), los cuales estimulan la neovascularización en la retina. (C) Fase 2: la retina hipóxica estimula la expresión de la eritropoyetina (EPO) y el factor de crecimiento endotelial vascular (VEGF), que estimulan la neovascularización retiniana. Las concentraciones del factor de crecimiento 1 similar a la insulina (IGF-1) aumentan lentamente desde concentraciones bajas después del parto prematuro hasta concentraciones lo suficientemente altas como para permitir la activación de las vias de VEGF. (D) El tratamiento para la resolución de la retinopatia se puede dar en la fase 1, aumentando el IGF-1 y limitando el oxigeno para evitar la supresión de VEGF. Además, eEGF se puede suprimir en la fase con EPO = eritropoyetina. $\omega-3$ PUFA $=\omega-3$ ácidos grasos poliinsaturados.

\section{CLASIFICACIÓN}

Con el objetivo de describir y estandarizar los hallazgos de las patologías de la retina, la Academia Americana de Pediatría (AAP) recomendó utilizar la clasificación internacional CIROP, la cual toma en cuenta 4 elementos:

\section{Zona de retina comprometida:}

"Zona I: corresponde al círculo de la retina más posterior cuyo centro es la papila y cuyo radio es el borde de la distancia de la papila a la fóvea.

- Zona II: corresponde al círculo concéntrico externo al anterior cuyo radio se extiende desde el límite de la zona I a la ora serrata (a las 3 horas en el ojo derecho y a las 9 horas en el ojo izquierdo) y a la zona cercana al ecuador anatómico a temporal.

- Zona III corresponde a la creciente de retina temporal anterior a la zona II."( González 2011) $(5,13)$.

\section{Estadio clínico de la enfermedad:}

1.-Es evidente la línea de demarcación plana y blanquecina en el límite entre la retina vascular y avascular. El proceso de crecimiento de los vasos retinianos se ha detenido y se observa bifurcaciones anormales de los vasos retinianos terminales.
2.-La línea demarcatoria entre la retina vascular y avascular adquiere volumen y es similar a un rodete blanquecino, conocido como ridge. Los vasos retinianos se dilatan y bifurcan antes de llegar al ridge.

3.-El tejido proliferativo fibrovascular extra retinal se proyecta sobre el ridge y de éste hacia el vítreo o sobre el plano de la retina.

4.-Corresponde al desprendimiento traccional de retina parcial; asociado por lo general a un componente exudativo que puede o no comprometer la mácula.

5.-Referido al desprendimiento de retina total que puede llevar a la ceguera $(5,13,14)$.

\section{Extensión de la enfermedad}

Para esto se divide el fondo de ojo siguiendo las agujas del reloj.

\section{Presencia de enfermedad plus:}

Descrita como el aumento de la dilatación venosa y la tortuosidad arteriolar de los vasos del polo posterior, en al menos dos cuadrantes de la retina. Mientras que la enfermedad umbral es la presencia de cinco o más horas continuas $u$ ocho horas acumuladas de enfermedad en estadio 3 en zona I o II en presencia de enfermedad plus $(5,14)$. 


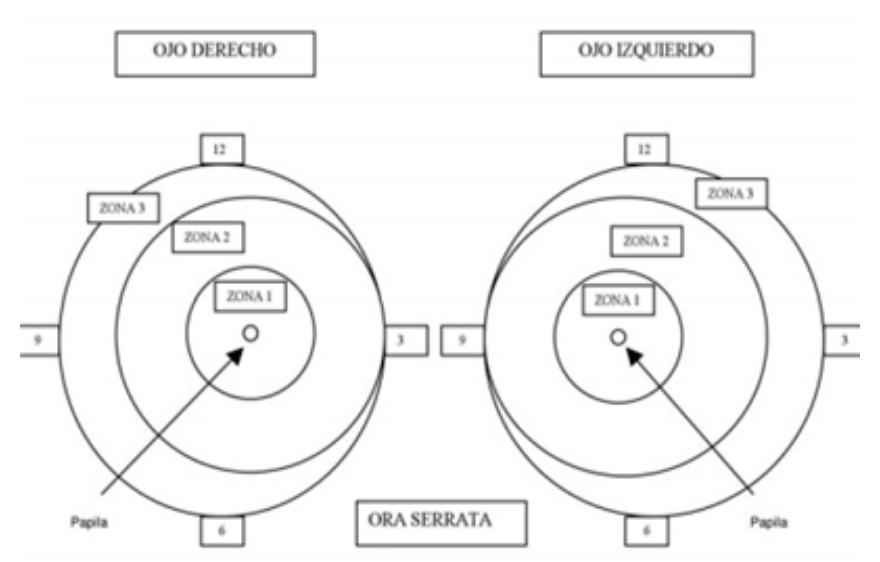

Figura $N^{\circ}$ 02. Clasificación de ROP (29).

Paris E, Sánchez I, Beltramino D, Copto A. Meneghello Pediatria. 6ta edición. Argentina: Editorial Médica Panamericana; 2013 (29).

\section{EPIDEMIOLOGÍA}

La retinopatía de la prematuridad afecta principalmente a los recién nacidos prematuros menores de $1500 \mathrm{~g}$ de peso al nacer o menores de 32 semanas de edad gestacional, sin embargo, casos inusuales se han descrito en los bebés de mayor peso al nacer que han tenido que recibir oxígeno $(16,17)$. La retinopatía del prematuro es mayormente prevenible y de buen pronóstico en los niños que reciben una cuidadosa administración de oxígeno, un diagnóstico oftalmológico temprano y un tratamiento oportuno (16).

En los países desarrollados, la retinopatía del prematuro afecta a niños menores de $1000 \mathrm{~g}$ de peso al nacer y con muy baja edad gestacional y no se registran casos en los de más de $1250 \mathrm{~g}$ de peso al nacer, ni casos inusuales (18). La administración cuidadosa de oxígeno para mantener saturaciones bajas (86-93\%) ha logrado disminuir la incidencia de retinopatía del prematuro de $35 \%$ a $13 \%$ y la necesidad de tratamiento del $7 \%$ al $1 \%$. Las actuales recomendaciones de tratamiento en estadios más precoces de la enfermedad (estadio pre-umbral), con el objetivo de mejorar el pronóstico visual, han aumentado levemente el número de niños tratados (de $1,3 \%$ a $3,5 \%)$. $(18,19)$.

En los países en vías de desarrollo, la enfermedad se presenta con características diferentes, afecta hasta al $34 \%$ de los prematuros con menos de $1500 \mathrm{~g}$ de peso al nacer, de los cuales $6-27 \%$ requerirán tratamiento. Se observan, además, niños con mayor peso al nacer y edad gestacional al nacer (903-1525 g y entre 26-33 sem) (20). A nivel mundial se estima que 60,000 niños se quedan ciegos debido a la enfermedad, de estos 25,000 (42\%) viven en América Latina. Con los avances de la neonatología que ha permitido la sobrevida de niños cada vez más pequeños, se estima que estos problemas se incrementarán $(19,20,21)$.

La incidencia varía de acuerdo al nivel de desarrollo socioeconómico de los países. Según reportes de estudios internacionales más del $50 \%$ de los pacientes lactantes con ceguera por retinopatía en el mundo están en Latinoamérica. Un $25 \%$ en menores de 1,500 gr o de 32 semanas se describen en Chile, Argentina y Brasil. Otro estudio en Latinoamérica reportó incidencia hasta del $82 \%$ en Nicaragua, $71 \%$ en Chile, $70 \%$ en Perú, $62 \%$ en Brasil y Colombia, $49 \%$ en Guatemala, $28 \%$ en México, $26 \%$ en Cuba y Argentina y $14 \%$ en Bolivia. Afortunadamente entre el 80 a $90 \%$ de los bebés diagnosticados con retinopatía de la prematuridad evolucionan espontáneamente $(20,21)$.

\section{FACTORES DE RIESGO}

La literatura describe los siguientes factores de riesgo. Peso del Recién Nacido y Edad Gestacional

El bajo peso al nacimiento como una edad gestacional temprana se han asociado con la frecuencia en la presentación de ROP, ya que están ligadas al desarrollo vascular de la retina y a la resistencia de la retina a episodios que podrían dañarla (22). Se ha descrito que ROP es más frecuente en aquellos bebés con peso al nacimiento igual o menor a 1500 gramos (23). Por otro lado, la edad gestacional promedio reportada con mayor frecuencia de presentar retinopatía en los prematuros es de 26.4 semanas $(24,25)$.

\section{Oxigeno Terapia}

La administración de oxígeno suplementario al recién nacido es un tema hasta ahora controversial, y es considerado uno de los principales factores de riesgo de ROP. La suplementación de oxígeno es muy importante en los periodos postnatales para prevenir la muerte de bebés prematuros, sin embargo el tiempo de administración debe ser el adecuado porque, las últimas investigaciones coinciden que la exposición prolongada podrían causar perdida de vasos en la ROP. La fluctuación de oxígeno suplementario hipoxia e hiperoxia en los niños se correlaciona con ROP según lo reportado, en donde presenta niveles elevados y en modelos de animales es peor que la hiperoxemia estable $(26,27)$.

\section{Transfusión Sanguínea}

Algunas investigaciones demuestran que el recibir transfusión de paquete globular durante las primeras cuatro semanas de vida, originará en el recién nacido un $30 \%$ de probabilidad de presentar ROP $(28,29)$. Por otro lado, también se ha cuestionado la administración de eritropoyetina humana recombinante (EPO) indicando que a través de sus propiedades angiogénicas, estimularía la proliferación de la vasculatura retiniana. Los estudios sugieren profundizar en hallar la relación entre la suplementación de hierro y la presentación de $\operatorname{ROP}(28,29,30)$.

\section{Comorbilidades}

Se han descrito diversas situaciones patológicas, aunque no concluyentes, que concurren con frecuencia en el recién nacido pre-término, tales como: disfunción neurológica, escaso crecimiento cerebral, anemia, transfusiones, insuficiencia respiratoria, apnea, displasia 
broncopulmonar, hemorragias intraventriculares, situaciones de acidosis y enterocolitis necrotizante (10, 31,32).

\section{Factores Genéticos}

Algunos estudios encuentran que los casos más frecuentes ocurren en los recién nacidos prematuros de raza blanca, así como los bebes de sexo masculino. Sin embargo, se recomienda recurrir a la genómica para lograr la identificación de los genes relevantes en esta patología $(27,33,34)$.

\section{Embarazo Múltiple}

En los embarazos múltiples, cada bebé recibe diferente flujo vascular intrauterino, generando que la vascularización retiniana también se produzca de manera desigual, situación que afecta directamente al recién nacido con menor peso. Es decir, el bebé afectado presentará una mayor área de la retina sin vascularizar. Los gemelos de igual edad gestacional no reciben la misma nutrición ni el mismo flujo vascular intrauterino, esto determina una desigual vascularización retiniana. Uno de los gemelos suele presentar un área de retina mayor sin vascularizar, habitualmente es el recién nacido de menor peso $(34,35)$.

\section{Terapia con surfactante}

Los recién nacidos prematuros con problemas respiratorios como síndrome de distress respiratorio reciben tratamiento con sustancia surfactante, lo cual mejora su esperanza de vida. Sin embargo, la terapia con surfactante como factor de riesgo de ROP tiene resultados ambiguos, algunos estudios no encuentran asociación significativa entre la terapia profiláctica con sustancia surfactante, mientras otras investigaciones sugieren que los pacientes con Síndrome de Distress Respiratorio tienen un alto riesgo de ROP por peso y edad gestacional al nacimiento $(35,36,37)$.

Restricción del crecimiento intrauterino (RCIU).

La restricción de crecimiento intrauterino (RCIU) está referido a la situación que provoca un peso neonatal por debajo del percentil 10 para la edad gestacional. Se describe como una reducción en el patrón de crecimiento fetal esperado, siendo el resultado de un crecimiento reducido innato o causado por otros efectos adversos ocasionados al feto (38). La literatura reporta una asociación altamente significativa entre la restricción del crecimiento intrauterino (RCIU) y la retinopatía en recién nacidos prematuros, constituyendo un factor de riesgo $(38,39)$.

\section{DISCUSIÓN}

La retinopatía de la prematuridad es una patología conocida desde los años 80 y que debido a los avances de la neonatología, que previene el fallecimiento de bebés prematuros, ha venido en incremento, reportándose en la actualidad una incidencia entre 19 a $30 \%$ a nivel mundial $(20,40,41)$.

Un estudio realizado en Ecuador durante el periodo 2010 al 2015, determinó un $20 \%$ de frecuencia de ROP en prematuros menores o igual a $1500 \mathrm{gr}$ de peso y menor o igual 33 semanas de gestación, reportando como principales factores asociados el sexo femenino y un mayor tiempo de estancia hospitalaria (> 30 días) (13).Mientras que otro estudio en neonatos con edad gestacional entre 27 y 34 semana, halló grado 1 retinopatía más frecuentemente, zona III de ambos ojos. Entre los factores de riesgo se mencionan: apnea, enterocolitis necrotizante, persistencia del conducto arterioso, sepsis, hemorragia intracraneana. El 1,2\% de los pacientes con retinopatía del prematuro recibieron tratamiento con láser y el $100 \%$ de ellos no presentaron ninguna secuela como complicación de la retinopatía del prematuro (42).

Un estudio revisión sistemática de niños nacidos con peso menor o igual a 1750 gramos encontraron con factores de riesgo la edad gestacional igual o inferior a 30 semanas y peso al nacimiento igual o menor a 1300 gramos, otros factores de riesgo son la no ganancia de peso, anemia y transfusión sanguínea, sepsis temprana y nosocomial, mientras que en menor proporción apnea, aplicación de surfactante, administración de esteroides prenatales. Mencionando además que el déficit visual y la ceguera son las secuelas encontradas en los pacientes con retinopatía severa (43).

Por otro lado, un estudio prospectivo transversal desarrollado en Colombia encontró asociación significativa entre el grado de retinopatía y algunas variables maternas, tales como: ruptura prematura de membranas ovulares, primigestantes, escolarización incompleta y domicilio rural. Mientras que entre las características clínicas del recién nacido se halló asociación significativa entre la presentación de retinopatía de la prematuridad con la fototerapia, neumonía neonatal, la asfixia perinatal, el tiempo de oxígeno-terapia mayor de 14 días y la ventilación mecánica mayor de tres días. Una de sus principales conclusiones sugiere que el bajo nivel socioeconómico y cultural de las madres podría asociarse con la presencia y grado de retinopatía del prematuro y las condiciones clínicas que conllevan a mayor exposición a oxigenoterapia (44).

A nivel nacional, se conoce que la incidencia detectada en el año 2013 fue de $28.2 \%$ no encontrando asociación significativa con el antecedente materno de preeclampsia (45). Mientras que estudios realizados sobre la retinopatia del prematuro en el año 2013 en Trujillo y otro en Chiclayo durante el año 2014 concluyeron que existen multifactores, tanto maternos como del neonato, que influyen en la presentación de la retinopatía del prematuro. La frecuencia de los factores maternos más frecuentes hallaron las infecciones del tracto urinario $(44,1 \%)$, preclampsia $(28,7 \%)$, eclampsia (20,6\%), hypertension arterial (19, 9\%), anemia y diabetes mellitus $(18,4 \%)$. 
$Y$ en los factores neonatales más frecuentemente asociados reportaron apnea (89,7\%), asfixia (78,7\%), sepsis neonatal (71,3\%), enfermedad de membrane hialina $(67,6 \%)$, peso al nacer menor de $1000 \mathrm{gr}$, edad gestacional menor de 30 semanas, oxigenoterapia mayor a 15 días,anemia con necesidad de transfusión, síndrome de dificultad respiratoria y acidosis metabólica $(46,47)$.

Una investigación retrospectiva realizada en el Hospital Nacional Arzobispo Loayza, reportada en Lima en el año 2015 concluyó que los factores de riesgo estadísticamente significativos asociados al desarrollo de ROP fueron edad gestacional menor de 28 semanas, peso al nacer menor de $1000 \mathrm{~g}$, oxigenoterapia, uso de ventilador mecánico, tiempo de exposición de oxígeno mayor de 15 días, uso de surfactante pulmonar, persistencia del conducto arterioso, síndrome de distress respiratorio, sepsis neonatal y el uso de hemoderivados (48).

\section{CONCLUSIONES}

Debido a los avances en el control de las condiciones de los neonatos prematuros, la presentación de retinopatía de la prematuridad ha incrementado a nivel mundial y con esto el entendimiento de su fisiopatología. Las investigaciones actuales no solo estudian el desarrollo patológico de la angiogénesis, sino que abordan el rol de las interacciones neurovasculares. Los factores de riesgo descritos a nivel mundial son bajo peso al nacer y edad gestacional, sin embargo los factores asociados que se vienen estudiando últimamente son los relacionados al tratamiento con oxígeno y su tiempo de exposición. Las comorbilidades también juegan un factor importante, principalmente la enterocolitis necrotizante, sepsis y la hemorragia intraventricular. Si bien hay estudios en Latinoamérica, así como algunos reportes en nuestro país, es necesario la realización de estudios longitudinales que incluyan los factores de riesgo, las características clínicas del bebé y de la madre, el procedimiento clínico y las características sociales para tener un mejor evidencia de la realidad de nuestro entorno, y en base a eso poder recomendar y/o mejorar los procedimientos clínicos de prevención y tratamiento en los departamentos de neonatología. 


\section{REFERENCIAS BIBLIOGRÁFICAS}

1. Baños OL, Toledo $Y$, Soto $M$, Mier M, Rúa R, Lapido $S$. Comportamiento de la retinopatía de la prematuridad en la provincia La Habana. Revista Cubana de Oftalmología. Cuba 2013; 26(2): 294-306.

2. Kavurt S, Ozcan B, Aydemir O, Bas AY, Demirel N. Risk of retinopathy of prematurity in small for gestational age premature infants. Indian Pediatr. 2014; 51(10):804-6.

3. Soto $M$, Mier $M$, Rúa $R$, López $M$, Toledo $Y$. Características clínicas epidemiológicas de la retinopatía de la prematuridad en recién nacidos de embarazos múltiples. Revista Cubana de Oftalmología. Cuba 2013; 26(1): 121-128.

4. Fariñas Z, Molina O, Guerra R, Rangel RA, Cabrera AC. Resultados del Programa de la retinopatía de la prematuridad en la Provincia de Villa Clara. Acta Médica del Centro. Cuba 2014; 8(3): 21-27.

5. Hartnett E, Penn JS. Mechanisms and management of retinopathy of prematurity. N Engl J Med. 2012; 367:2515-26.

6. Legrá Nápoles $S$, Ríos Araujo B, Dueñas Romeo B, López Fernández CR, Gutiérrez Escobar M, Lugones Sánchez J. Caracterización de la retinopatía de la prematuridad en neonatos con factores de riesgo. Medisur. 2011; 9:13-21.

7. Castro Pérez PD, Rodríguez Masó S, Rojas Rondón I, Padilla González C, Fernández Cherkásova L. Epidemiología y rehabilitación de la retinopatía de la prematuridad en el servicio de baja visión. Rev Cubana Oftalmol. 2010; 23:156-68.

8. Curbelo Quiñones Luvia, Durán Menéndez Raisa, Villegas Cruz Débora Mercedes, Broche Hernández Aimeé, Alfonso Dávila Antonio. Retinopatía del prematuro. Rev Cubana Pediatr. 2015; 87(1): 69-81.

9. Reyes C, Campuzano M, Pardo RV. Prevalencia de retinopatía en el prematuro. Archivos de Investigación Materno Infantil. México 2011, 3(3): 132-137.

10. Hellstrom A, Smith LE, Dammann O. Retinopathy of prematurity. Lancet. 2014; 382(9902):1445-57.

11. Rodríguez M, Concepción JA. Referentes teóricos sobre la retinopatía de la prematuridad y sus criterios de pesquisa. Gaceta Médica Espirituana. Cuba 2016; 18(2): 35-45.

12.12. Reddy MA, Patel HI, Karim SM, et al. Reduced utility of serum IGF-1 levels in predicting retinopathy of prematurity reflects maternal ethnicity. $\mathrm{Br} \mathrm{J}$ Ophthalmol. 2016 Apr; 100(4):501-4.

13. González J. Retinopatía del prematuro. Revista Médica de Costa Rica y Centro-América. Costa Rica 2011; 68(596): 45-48.

14. Ortega-Molina JM, Anaya-Alaminos $R$ Uberos-Fernandez J, Solans-Perez de Larraya $A_{\text {, }}$ Chaves-Samaniego MJ, Salgado-Miranda $A$, et al. Genetic and Environmental Influences on Retinopathy of Prematurity. Mediators Inflamm. 2015: 2015:764159.
15. Paris E, Sánchez I, Beltramino D, Copto A. Meneghello Pediatria. 6ta edición. Argentina: Editorial Médica Panamericana; 2013.

16. Hartnett ME. Advances in understanding and management of retinopathy of prematurity. Surv Ophthalmol. 2017; 62(3):257-76.

17. Shah PK, Prabhu V, Karandikar SS, Ranjan R, Narendran V, Kalpana N. Retinopathy of prematurity: Past, present and future. World J Clin Pediatr. 2016; 5(1):35-46.

18. Tan Z, Chong C, Darlow B, Dai S. Visual impairment due to retinopathy of prematurity (ROP) in New Zealand: a 22-year review. Br J Ophthalmol. 2015; 99:801-806.

19. Kusaka S. Current concepts and techniques of vitrectomy for retinopathy of prematurity. Taiwan J Ophthalmol. 2018; 8(4):216-21.

20. Carrion JZ, Fortes Filho JB, Tartarella MB, Zin A, Jornada ID, Jr. Prevalence of retinopathy of prematurity in Latin America. Clin Ophthalmol. 2011; 5:1687-95

21. Lomuto CC, Galina L, Brussa M, Quiroga A, Alda E, Benítez AM, et al. Epidemiología de la retinopatía del prematuro en servicios públicos de la Argentina durante 2008. Arch. Argent. Pediatr. Argentina 2010; 108(1): 24-30.

22. Lissauer T, Fanaroff AA. Neonatología. 2da edición. Argentina: Editorial Médica Panamericana; 2014

23. Moya EL. Retinopatía en el neonato prematuro nacido en el hospital La Caleta durante el 2014. Revista In Crescendo. Ciencias de la Salud. Perú 2015; 2(2): 500-509.

24. Cabrera AD, Fariñas Z. Características de la retinopatía de la prematuridad en Villa Clara. Acta Médica del Centro. Cuba 2014; 8(1): 1-7

25. Uwizihiwe F, Prevalence And Predisposing Factors Of Retinopathy Of Prematurity In Low Birth Weight Preterm Neonates At One District Of Rwanda: A Case Of Muhima District Hospital, Neonatal Unit. August 2016

26. Gutiérrez Cl, Vergara EF, Rojas $P$, Labrín C. Retinopatía de la prematuridad en el Hospital Regional Docente Las Mercedes. Chiclayo 2007-2009. Revista del Cuerpo Médico del Hospital Nacional Almanzor Aguinaga Asenjo. Perú 2012; 5(1): 30-32.

27. Ying GS, Bell EF, Donohue $P$, Tomlinson LA, Binenbaum G, Group GRR. Perinatal Risk Factors for the Retinopathy of Prematurity in Postnatal Growth and Rop Study. Ophthalmic Epidemiol. 2019:1-9.

28. Leng Y, Huang W, Ren G, Cai C, Tan Q, Liang Y, et al. The treatment and risk factors of retinopathy of prematurity in neonatal intensive care units. BMC Ophthalmol. 2018; 18(1):301.

29. Yang $Z$, Wang $H$, Jiang $Y$, Hartnett ME. VEGFA activates erythropoietin receptor and enhances VEGFR2-mediated pathological angiogenesis. 
Am J Pathol. 2014; 184(4):1230-9

30.Chávez B, Rojas OA. Antecedentes Perinatales para Retinopatía del Prematuro en el Hospital Infantil del Estado de Sonora. Boletín Clínico del Hospital Infantil del Estado de Sonora. México 2012; 29(2): 72-76.

31. Martínez MS, Acuña R, Monzón A, Murillo J. Incidencia y Características Clínicas en Pacientes con Retinopatía del Prematuro en el Hospital de la Mujer. Archivos de Salud de Sinaloa. México 2015; 7(3): 92-97.

32. Goldstein GP, Leonard SA, Kan P, Koo EB, Lee HC, Carmichael SL. Prenatal and postnatal inflammation-related risk factors for retinopathy of prematurity. J Perinatol. 2019.

33. Dwivedi A, Dwivedi D, Lakhtakia S, Chalisgaonkar C, Jain S. Prevalence, risk factors and pattern of severe retinopathy of prematurity in eastern Madhya Pradesh. Indian J Ophthalmol. 2019; 67(6):819-23.

34. Hansen RM, Moskowitz A, Akula JD, Fulton AB. The neural retina in retinopathy of prematurity. Prog Retin Eye Res. 2017; 56:32-57.

35.Zamorano CA, Salgado M, Velásquez B. Factores de riesgo asociados a retinopatía del prematuro. Gaceta Médica de México. México 2012; 148(1): 19-25.

36. Beharry KD, Valencia GB, Lazzaro DR, Aranda JV. Pharmacologic interventions for the prevention and treatment of retinopathy of prematurity. Semin Perinatol. 2016; 40(3):189-202.

37.Jivabhai Patel $S$, Bany-Mohammed F, McNally L, Valencia GB, Lazzaro DR, Aranda JV, et al. Exogenous Superoxide Dismutase Mimetic Without Scavenging H2O2 Causes Photoreceptor Damage in a Rat Model for Oxygen-Induced Retinopathy. Invest Ophthalmol Vis Sci. 2015; 56(3):1665-77.

38. Sharma D, Shastri S, Sharma P. Intrauterine Growth Restriction: Antenatal and Postnatal Aspects. Clin Med Insights Pediatr. 2016; 10:67-83

39.Jacinto RJ. Prevalencia y factores de riesgo para el desarrollo de retinopatía de la prematuridad en el servicio de neonatología del Hospital Sergio E. Bernales, 2014 [Tesis Pre-Grado]. Perú: Universidad Ricardo Palma. Facultad de Medicina Humana; 2016.

40.Azami M, Jaafari Z, Rahmati S, Farahani AD, Badfar G. Prevalence and risk factors of retinopathy of prematurity in Iran: a systematic review and meta-analysis. BMC Ophthalmol. 2018; 18(1):83.
41. Zimmermann MA, Rojas M, Galicia I. Características de pacientes con retinopatía del prematuro tratados con láser en el Instituto Guatemalteco de Seguridad Social del 2012 al 2014. Revista Mexicana de Oftalmología. Guatemala 2017; 91(6): 311-315.

42. Chiriboga KM. Prevalencia de la Retinopatía y los factores de riesgo en prematuros. Hospital San Francisco de Quito, octubre 2014-2016 [Tesis Post-Grado]. Ecuador: Universidad Central del Ecuador. Facultad de Ciencias Médicas; 2017.

43. López RY. Factores de riesgo asociados a retinopatía del prematuro en menor o igual a 1750 gramos al nacer, que requirió tratamiento con láser en Hospital de Niños Benjamín Bloom de enero 2012 a diciembre 2013 [Tesis Post-grado]. El Salvador: Universidad de El Salvador. Facultad de Medicina; 2015.

44. Vidal D, Lasso D, Ordoñez S, Acosta F, Merchán AM, Muñoz A, et al. Retinopatía del prematuro: Caracterización de la población y factores asociados. Revista Facultad Ciencias de la Salud. Universidad del Cauca. Colombia 2013; 15(4): 17-24.

45. Gayoso M, Genaro S, Sáenz S, Webb DV, Rospigliosi $\mathrm{ML}$, Rivera F. Pre-eclampsia como factor asociado al desarrollo de retinopatía en prematuros de muy bajo peso al nacer nacidos en el Hospital Nacional Cayetano Heredia entre Enero 2005 y Diciembre 2011. Revista Peruana de Pediatría. Perú 2013; 66(2): 84-94.

46. Guerrero MR, Sánchez SP, Lizárraga IG. Factores de riesgo que inciden en la retinopatía del prematuro en el servicio de neonatología en un hospital del ministerio de salud. Revista Salud y Vida Sipanense. Perú 2016; 3(1): 6-19.

47. Ynguil AM. Factores de riesgo de retinopatía de la prematuridad en niños con muy bajo peso al nacer [Tesis Post-Grado]. Perú: Universidad Nacional de Trujillo. Facultad de Medicina; 2013.

48. Cruz JE. Incidencia y factores de riesgo de retinopatía de la prematuridad en el servicio de neonatología del Hospital Nacional Arzobispo Loayza, periodo 2010-2014 [Tesis Pre-Grado]. Perú: Universidad Nacional de San Agustín. Facultad de Medicina; 2015.

\section{PANACEA}

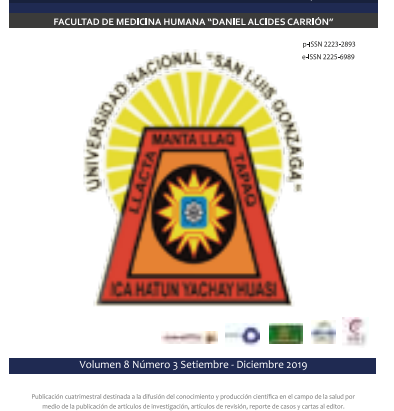

